\title{
Tumor ferroptosis status demonstrated vulnerability to chemotherapy and reflected immune-activation in colorectal cancer
}

\section{Yang Lv}

Zhongshan Hospital Fudan University

\section{QingYang Feng}

Zhongshan Hospital Fudan University

\section{ZhiYuan Zhang}

Zhongshan Hospital Fudan University

\section{Peng Zheng}

Zhongshan Hospital Fudan University

\section{DeXiang Zhu}

Zhongshan Hospital Fudan University

\section{YiHao Mao}

Zhongshan Hospital Fudan University

\section{YuQiu Xu}

Zhongshan Hospital Fudan University

\section{MeiLing Ji}

Zhongshan Hospital Fudan University

JianMin Xu

Zhongshan Hospital Fudan University

GuoDong He ( $\square$ angelhgd@163.com )

Zhongshan Hospital Fudan University

\section{Research}

Keywords: Colorectal cancer, Immune response, Ferroptosis, Chemotherapy

Posted Date: October 2nd, 2020

DOl: https://doi.org/10.21203/rs.3.rs-84128/v1

License: (c) (1) This work is licensed under a Creative Commons Attribution 4.0 International License.

Read Full License 


\section{Abstract}

Background: Existing studies for ferroptosis and prognosis in colorectal cancer (CRC) were limited. In this study, we aim to investigate the prognostic role of ferroptosis markers in patients with CRC and exploration of its micro-environmental distributions.

Methods: A total of 911 patients from 2008 to 2013 with CRC were enrolled. Immunohistochemical staining was performed for CRC patients' tissue microarray. Selection and prognostic validation of markers were based on mRNA data from the cancer genome atlas (TCGA) database. Gene Set Enrichment Analysis (GSEA) was performed to indicate relative immune landmarks and hallmarks. Ferroptosis and immune contexture were examined by CIBERSORT. Survival outcomes were analyzed by Kaplan-meier analysis and cox analysis.

Results: A panel of 42 genes was selected. Through mRNA expression difference and prognosis analysis, GPX4, NOX1 and ACSL4 were selected as candidate markers. By IHC, increased GPX4, decreased NOX1 and decreased FACL4 indicate poor prognosis and worse clinical characteristics. Ferroptosis score based on GPX4, NOX1 and ACSL4 was constructed and validated with high C-index. Low ferroptosis score can also demonstrate the better progression free survival and better adjuvant chemotherapy (ACT) responsiveness. Moreover, tumor with low ferroptosis score tend to be infiltrated with more CD4+ T cells, CD8+ T cells and less M1 macrophage. Finally, we found that IFN- $\gamma$ was potentially the central molecule at the crossroad between ferroptosis and onco-immune response.

Conclusion: Ferroptosis plays important role on CRC tumor progression, ACT response and prognosis. Ferroptosis contributes to immune-supportive responses and IFN- $\gamma$ was the central molecule for this process.

\section{Background}

Colorectal cancer (CRC) is common around the world $(1,2)$. In China, CRC ranks the third most frequently diagnosed malignancy and third leading cause of cancer-associated mortality(3). Unfortunately, even after radical excision and subsequent systematic adjuvant chemotherapy (ACT), there were still 15\%-25\% CRC patients suffering from systemic recurrence (including local recurrence and distant metastasis)(4). Detailed stratification for prognosis and treatment responsiveness of CRC still need further exploration.

Ferroptosis is a newly-recognized form of necrotic cell death marked by oxidative modification of membranes via an iron-dependent mechanism(5). Publications indicated potential role of ferroptosis on cancer translational medicine(6), including overcoming chemotherapy resistance(7) and progression prevention(8). However, clinically, relations between ferroptosis and CRC prognosis were still lacking.

Here, through candidate markers screen, three ferroptosis markers (GPX4, NOX1 and ACSL4) were selected. And we explored the clinical prognostic value of GPX4, NOX1 and ACSL4. Furthermore, a novel 
ferroptosis score based on GPX4, NOX1 and FACL4 were constructed and validated. Finally, immune micro-environemental influences brought by ferroptosis were also explored.

\section{Methods}

\section{Patient eligibility and follow-up principle}

This study retrospectively enrolled consecutive 911 patients from Colorectal cancer center, Zhongshan Hospital, Fudan University (Shanghai, China) between 2008 to 2012. Of 911 patients, 528 were males. For construction and validation of nomogram, patients were randomly divided into training set (455 patients) and validation set (456 patients). Postoperative ACT was administrated to patients according to the Chinese, NCCN CRC guidelines(9-11) and patients' will. This study was approved by the Ethical Committee of Zhongshan Hospital, Fudan University. Follow-up principles were based on the Chinese guideline for colorectal cancer $(10,12)$.

\section{Ferroptosis Marker Selection}

To determine ferroptosis markers list, an unbiased search for relevant articles was done on Pubmed for all full-text articles pertaining to ferroptosis. Studies were identified using the term "cancer" OR "tumor" OR "neoplasm" AND "ferroptosis". Details were shown in supplementary information.

\section{Tcga Data Source And Processing}

Raw data of RNA sequence and matched clinical characteristics of colon and rectal cancer were downloaded from the online database The Cancer Genome Atlas (https://tcga-data.nci.nih.gov/tcga/). It contains 51 normal tissues and 647 tumor tissues. Significant up and down-regulated genes were defined as fold change of at least $1.5 \mathrm{X}$ and adjusted P-value $\leq 0.05$. The results were visualized as a heat-map plot using ggplot2 (RRID:SCR_014601) package(13). For significant different markers, prognostic value of each gene on CRC was determined for further markers screening.

\section{Immunohistochemistry And Intensity Evaluation}

Formalin-fixed paraffin-embedded surgical specimens were used for tissue microarray (TMA) construction and subsequent immunohistochemistry (IHC) study as described previously $(14,15)$. Histological review was also conducted to avoid necrotic and hemorrhagic tumor regions.

The immunoreactivity for GPX4, NOX1 and FACL4 in cancer cells was calculated as the product of two independent scores, the proportion of positive tumor cells in the tissues and the average intensity of positive tumor cells in the tumor tissues(16). The CD4-positive T cell, CD8-positive T cell and CD86positive M1 macrophage infiltration was recorded as the mean number of tryptase-positive/HPF from three randomized fields(15). The expression was scored independently by two pathologists who were blinded to clinical pathological characteristics. Cut-off was determined as median score.

\section{Gene Set Enrichment Analysis}


Gene set enrichment analysis (GSEA) was performed by the GSEA desktop application v.3.0 with 1,000 permutations(17). Molecular Signatures Database (MSigDB) v6.0, was applied as a reference to determine pathways differentially enriched between low and high mRNA expression groups(17).

\section{Statistics}

Statistical analyses were performed using the SPSS statistical package (22.0; SPSS; RRID:SCR_002865), R studio (R Project for Statistical Computing, RRID:SCR_001905) and prism 6 (GraphPad Prism, RRID:SCR_002798). NOX1, GPX4 and FACL4 expression between normal and cancer tissues was compared by paired Wilcoxon signed rank test. The correlations between continuous valuables were analyzed using Spearman rank correlation test and $x^{2}$ test. Time-dependent cut-off values were determined when positive likelihood ratio (PLR) were the largest one. PFS and OS analyses were carried out using the Kaplan-Meier method and results were compared using a log-rank test. A multivariable Cox proportional hazards model predicting OS was performed using backward stepwise selection. Nomogram was constructed based on R studio (rms package). Risk factors were expressed as the hazard ratio [HR, 95\% confidence interval $(\mathrm{Cl})$ ]. Statistical significance was defined as P-value less than 0.05 .

\section{Results}

\section{Identification of prognostic ferroptosis markers in CRC}

Ferroptosis-related genes were selected according to publications(18) and were shown in Table S1. KEGG (Fig. 1A) and GO analysis (Fig. 1B) and Protein-Protein interaction (PPI) network (Figure S1) of these genes were constructed to validate the biological relation with ferroptosis. Differential mRNA expression of these genes were constructed in Fig. 1B and ranked by log fold changes (FC). Further Kaplan-meier analysis of first 15 genes $(P<0.01)$ and last 8 genes $(P<0.01)$ were performed to determine prognostic role. In Fig. 1C, NOX1 low expression $(P=0.013)$, GPX4 high expression $(P=0.008)$ and ACSL4 low expression $(P=0.048)$ were separately regarded as risk factors for $C R C$ patients' prognosis. Prognosis analysis of the other 16 genes were shown in Figure S2A (up-regulated genes) and Figure S2B (downregulated genes).

\section{Correlation Between Ferroptosis Markers And Clinical Characteristics In Crc}

Protein expression of GPX4, NOX1, and FACL4 (ACSL4) were identified by IHC staining. Representative images were shown in Fig. 2 (A, B and C). GPX4, NOX1 and FACL4 expression were higher than paired normal tissues (Figure S3A), which was further validated through Gene Expression Profiling Interactive Analysis (GEPIA) (Figure S3A)(19). Clinical correlation between these markers and baseline clinical characteristics was shown in Table S2. We found that higher expression of GPX4, lower expression of NOX1 and FACL4 indicated larger primary tumor size $(P=0.001)$. Separately, higher expression of GPX4 were clinically correlated with higher lymph node metastasis $(P=0.029)$, lower NOX1 correlated with higher tumor invasion stage $(P=0.001)$ and lower FACL4 indicated more distant metastasis $(P=0.001)$, these were all demonstrated in Fig. 2(D, E and F). 


\section{Prognostic Role Of Ferroptosis Markers In Crc}

To investigate clinical value of three ferroptosis markers in CRC, we performed Kaplan-Meier survival analyses and Log-rank tests were applied to evaluate prognostic merit in all CRC patients. As were shown in Fig. 2 (G, H and I), low expression of GPX4 (P<0.001; 95\% Cl: 0.54-0.84; HR:0.68), high expression of NOX1 (P<0.001, 95\% Cl: 1.20-1.85; HR:1.49) and high expression of FACL4 ( $<<0.001,95 \%$ Cl: 1.121.75; HR:1.47) demonstrated better prognosis in patients with CRC. Univariate and Multivariate analysis were shown in Table S3, expression of GPX4 ( $P=0.014,95 \% \mathrm{Cl}$ : 0.58-0.94; HR: 0.74), NOX1 ( $P=0.026$, 95\% Cl: 1.03-1.67; HR:1.31) and FACL4 ( $P=0.015,95 \%$ Cl: 1.21-1.66; HR:1.34) were independent factors for OS in CRC. Event-based ROC curves for OS were constructed based on GPX4, NOX1 and FACL4, respectively. The AUC were separately 0.57 for GPX4 (Figure S4A), 0.53 for NOX1 (S4B) and 0.56 for FACL4 (S4C). Furthermore, time dependent ROCs were constructed to determine the prognostic role of

ferroptosis-related markers, Figure S4 indicated survival dependent AUCs of GPX4 (S4D), NOX1 (S4E) and FACL4 (S4F) for OS.

\section{Subgroup analysis of GPX4, NOX1 and FACL4 for OS in CRC}

In subgroup analysis, patients were divided into four groups according to AJCC stage: stage I, stage II, stage III and stage IV. In stage I CRC (Fig. 3A), expression of GPX4 ( $P=0.87, \mathrm{HR}: 1.07,95 \% \mathrm{Cl}$ : 0.39-2.95) and FACL4 ( $P=0.48$, HR: $1.43,95 \% \mathrm{Cl}: 0.52-3.98)$ have no prognostic role for $\mathrm{OS}$, while patients with low level of NOX1 had worse survival outcomes ( $P=0.03$, HR: $1.07,95 \% \mathrm{Cl}: 0.39-2.95)$. In stage II CRC (Fig. 3B), expression of three individual marker demonstrated no significant prognostic role $(P>0.05)$. And in stage III and stage IV CRC, GPX4, NOX1 and FACL4 were regarded as significant prognosis-related factors (all $P<0.05$, Fig. 3 C and 3D).

\section{Ferroptosis Score For Prognosis In Crc: Development And Validation}

Correlation between three ferroptosis markers was performed through linear regression analysis. IHC Expression relations were determined among GPX4, NOX1 and FACL4 in Fig. 3E, 3F and $3 G(P<0.05)$. To assess the comprehensive ferroptosis status, all 911 patients were divided randomly into 2 cohorts (training cohort and validation cohort). Baseline characteristics were shown in Table S4 and there was no difference between training cohort and validation cohort. clinical model incorporating GPX4, NOX1 and FACL4 was constructed based on characteristics of training cohort. Figure $3 \mathrm{H}$ conferred the nomogram for CRC prognosis and the calibration curves were presented high agreement between predicted survival and actual survival in both training cohort (Figure S5) and validation cohort (Fig. 3I). Furthermore, C-index combining ferroptosis score with TNM staging yield higher accuracy than TNM alone in both sets (Table S5). Subgroup analysis for different TNM stage were shown in Figure S6.

\section{Ferroptosis score as a predictive parameter for tumor progression in CRC}

Kaplan-meier method was used for PFS analysis. For all patients, individual GPX4 and FACL4 expression has no role on DFS stratification ( $P>0.05$ ), while expression of NOX1 had statistical correlation with PFS 
$(\mathrm{P}<0.05)$. Details were shown in Fig. 4A to 4C (IHC findings) and Figure S7 (TCGA findings). Ferroptosis score was further divided into three groups: high score group (14-22 scores), medium score group (7-14 scores) and low score group (0-7 scores). Consistent with our identification, low-score group patients showed the most favorable PFS, while high score group patients demonstrated worst PFS (Fig. 4D). Furthermore, we performed Cox proportional hazards regression analysis to assess the relationship among different groups. As was shown in Fig. 4E, ferroptosis-based risk classification model could be regarded as an independent prognostic factor to predict recurrence for CRC patients.

\section{Ferroptosis score and ACT in stage II and III CRC}

Furthermore, we sought to the discover whether different risk groups indicated distinct responsiveness to ACT in CRC patients. For stage IV CRC patients, not all patients underwent radical resection for metastases and treatment regimen often incorporated targeted therapies. To be more precise, we focused on ACT benefit in stage II and III patients. Results indicated ferroptosis score could be used to stratify patients into different risk subgroups, low and median ferroptosis score patients benefited more from ACT and had better PFS (Fig. 4F) and OS (Fig. 4G) (P<0.001). In contrast, high score patients had inferior therapeutic responsiveness to ACT. Details for PFS and OS between ACT and no-ACT cohorts were shown in Figure S8.

\section{Elements of ferroptosis score system shape immune contexture in CRC}

By using GSEA to compare the mRNA expression profile between low and high GPX4, NOX1 and ACSL4, respectively. SsGSEA analysis demonstrated changes of immune contexture brought by the ferroptosis markers (Fig. 5A-C, left part). Multiple immune-related pathways were found, including CD4 + T cells (Fig. 5A, middle part), CD8 T cells (Fig. 5B, middle part) and M1 macrophage (Fig. 5C, middle part). As was shown in Fig. $5 \mathrm{~A}, \mathrm{CD} 4+\mathrm{T}$ cells pathway were significantly enriched in low GPX4 groups with the Normalized Enrichment Score (NES) of $-2.06(P=0.000)$, these results were validated by CIBERSORT analysis which indicated GPX4 expression was significantly negatively correlated with CD4 + T cell infiltration (Fig. 5A, right part). Also, expression correlations between $\mathrm{M} 1$ macrophage and NOX1(NSE=-2.41, P = 0.000), CD8 + T cell infiltration and ACSL4 (NSE $=2.08, P=0.000$ ) were indicated through GSEA and CIBERSORT analysis (Fig. 5B and Fig. 5C).

Given the above findings, IHC staining of CD4, CD8 and CD86 were further performed for validation. Representative images of double staining were shown in Fig. 5D. Analysis demonstrated that GPX4 expression was negatively correlated with $C D 4+T$ cell infiltration $\left(R^{2}=0.16, P=0.01\right)$, NOX1 expression was negatively correlated with $M 1$ macrophage infiltration $\left(R^{2}=0.32, P<0.001\right)$ and FACL4 expression was positively correlated with $C D 8+T$ cell infiltration $\left(R^{2}=0.16, P=0.002\right)$. Details were shown in Fig. $5 E$.

\section{IFN- $\gamma$ were potentially involved in tumor ferroptosis and indicated better prognosis in CRC}

By GSEA for Hallmarks, interferon- $\gamma$ (IFN- $\gamma$ ) response were all significantly enriched for all three markers (low GPX4, low NOX1 and high ACSL4) (Fig. 5F). The NES were separately - 2.01, -2.48 and 1.76 for 
GPX4, NOX1 and ACSL4. GPX4 expression and NOX1 expression were negatively correlated with IFN-y (P $<0.001)$, while ACSL4 were positively correlated with IFN-y $(P<0.001)$.

To further validate the results, JAK expression and Stat1 expression was evaluated. As was demonstrated in Fig. 5G, GPX mRNA expression was negatively correlated with JAK $\left(R^{2}=0.23, P<0.001\right)$ and Stat $1\left(R^{2}\right.$ $=0.10, P<0.001)$, NOX1 mRNA expression was negatively correlated with JAK $\left(R^{2}=0.08, P<0.001\right)$ and Stat1 $\left(R^{2}=0.09, P<0.001\right)$ and ACSL4 mRNA expression was positively correlated with JAK $\left(R^{2}=0.23, P\right.$ $<0.001)$ and Stat1 $\left(R^{2}=0.18, P<0.001\right)$. All these results were consistent with GSEA findings and indicated ferroptosis score were highly correlated with anti-tumor immunity and IFN- $\gamma$ may be the key regulator at the crossroad between ferroptosis and anti-tumor immunity. Furthermore, based GEPIA cohort analysis, high expression of IFNG were statistically demonstrated with longer duration of DFS ( $P=$

\subsection{8) (Figure S9).}

\section{Discussion}

Ferroptosis is a newly recognized cell death modality distinct from other forms of cell death $(20,21)$. Researchers have reported that ferroptosis could be triggered by diverse physiological conditions and pathological stimulus(21). Since the discovery of ferroptosis, targeting ferroptosis has been regarded as a novel anti-cancer strategy(22), compelling evidence indicated that compounds like erastin and sorafenib could induce tumor ferroptosis(23). Besides, potential molecular mechanisms involved in ferroptosis were observed in many experimental cancer models(24-26), including inactivation of GPX4, up-regulation of FACL4. However, given the promising opportunities and challenges, in CRC, relevance between ferroptosis and clinical characteristics was still little known. Figure 6A demonstrated diagram for this study and Fig. 6B conferred to the prognostic role of ferroptosis score and the potential mechanisms.

Tumor ferroptosis is a complex process. This process can be modulated through many pathways and communicated by other microenvironment cells(27). Given the close relation between ferroptosis and cell metabolism, truly CRC ferroptosis status may not be reflected by single molecule. Thus through transcript difference analysis and prognosis screen, a small panel including GPX4, NOX1 and ACSL4 was selected for further study.

Realizing proteins as the "executioners of life" that determine phenotype, IHC staining for GPX4, NOX1 and FACL4(ACSL4) were performed on TMA and recorded by independent pathologist. Results from TMA were reported to be same robustness as classic tumor Sect. (28). In our study, prognostic value of protein level expression was highly consistent with mRNA level expression. Low expression of GPX4 indicated better survival, high expression of NOX1 and FACL4 demonstrated better survival in CRC. Characteristics were also included to compare the clinical relevance. High GPX4 expression was significantly correlated with lymphnode metastasis $(P=0.029)$, tumor with low NOX1 expression tend to be higher pathological T stage $(P=0.001)$ and low expression of FACL4 was statistically correlated with higher $M$ stage, besides, all the three markers expression were clinically correlated with primary tumor size (all $P=0.001)$. 
We further determine AUC of OS for each marker. AUC of GPX4, NOX1 and FACL4 were separately merely $0.57,0.53$ and 0.56. Survival time-dependent AUC was also not more than 0.65 . These results indicated again that single ferroptosis marker was not enough to reflect the real ferroptosis status. We wonder whether combining GPX4, NOX1 and FACL4 could complementarily present tumor ferroptosis status in CRC. In the next step, by randomly dividing our patients into two groups, a nomogram (ferroptosis score) based on COX multivariable analysis was first construed and validated. Besides, incorporating ferroptosis to classic TNM stage could effectively improve the prognosis prediction power on CRC. In the next step, applying ferroptosis score could also stratify patients into different tumor progression groups (PFS), analysis of ACT responsiveness demonstrated same result. Compared to high ferroptosis score cohort, CRC patients' tumor with low/medium ferroptosis score would be easier to benefit from ACT.

Down-regulation of GPX4(29, 30), up-regulation of ACSL4(31) and up-regulation of NOX1(32) facilitate ferroptosis sensitivity through different pathways. Potential mechanisms have been reported. However, so far, immune-modulation role of ferroptosis sensitive tumor cells was not revealed. It is reported that dying cells, mostly in the context of ferroptosis sensitivity, communicate with immune cells by a set of signals(33). This facilitate the immune cells to locate ferroptotic cells in the tissue and mediate the movement of immune cells within tissues. We first reported microenvironment influences brought by ferroptosis sensitive tumor cells. Based on GSEA analysis and CIBERSORT analysis, tumor cells with low GPX4 tend to be infiltrated by higher proportion of CD4 + T cell, expression of NOX1 was negatively correlated with M1 macrophage infiltration and ACSL4 was positively close to CD $8+T$ cell infiltration. To further validate these findings, double IHC staining of CD4 and GPX4, CD86 and NOX1, CD8 and FACL4 was performed and results confirmed the relations. Recently reports demonstrated that IFN- $\gamma$ produced by tumor infiltrating T cells contributed to tumor ferroptosis $(34,35)$. In our study, through cancer hallmarks analysis, IFN- $\gamma$ was regarded as a central molecule at the cross roads between ferroptosis and immune responses in CRC. Furthermore, downstream factors expression (JAK and Stat1) was validated and consistent with results of GSEA enrichment. This could be reasonable that higher infiltration of CD $4+T$ and CD8 + T cell resulted in higher expression of IFN- $\gamma$, which facilitate tumor ferroptosis and then patients' prognosis. On the other hand, reduced IFN-y could decrease the process of M0 Macrophages into $\mathrm{M} 1$ polarization(36), which was consistent to our results.

There are still some limitations. First, the gene list regarding ferroptosis-related markers was based on previous publications and there may be more precise and specific markers for CRC which was still uncovered yet. Second, to conduct a real world study, we enrolled consecutive patients to continue the study cohort. There were inevitable imbalances at the baseline, especially in the application of ACT. This imbalance could interfere with the results. But the predictive biomarker must face it in clinical applications. Third, for nomogram construction, the two cohorts of patients came from the same medical center, thus lacking of external validation. Finally, the central role of IFN- $\gamma$ was determined and validated through bioinformatics analysis. More detailed experimental works of molecular mechanisms are still required in the future. 


\section{Conclusion}

In summary, our study showed GPX4, NOX1 and ACSL4 were significant prognostic ferroptosis-related markers in CRC. Ferroptosis markers expression was closely related with clinical characteristics; ferroptosis score based on GPX4, NOX1 and FACL4 can effectively reflect CRC prognosis, tumor progression and ACT responsiveness with high C-index. Furthermore, tumor with low ferroptosis score may be infiltrated with more CD4 + T cells, CD $8+T$ cells and less M1 macrophage. In this process, IFN- $y$ may be potentially the central role at the crossroad between ferroptosis and onco-immune response.

\section{List Of Abbreviations}

CRC, colorectal cancer; TCGA, the cancer genome atlas; GSEA, Gene Set Enrichment Analysis; ACT, adjuvant chemotherapy; TMA, tissue microarray; IHC, immunohistochemical staining; MsigDB, Molecular Signatures Database; PLR, positive likelihood ratio; $\mathrm{Cl}$, confidence interval; PPI, protein-protein interaction; FC, fold change; GEPIA, Gene Expression Profiling Interactive Analysis; NSE, Normalized Enrichment Score; IFN- - , interferon- - .

\section{Declarations}

\section{Ethics approval and consent to participate}

Written informed consent was obtained by all the patients. The study protocol followed the ethical guidelines of the Declaration of Helsinki and was approved by the Ethical Committee of Zhongshan Hospital of Fudan University. The ethics approval ID was B2017-166R.

\section{Consent for publication}

We have obtained consent to publish from the participant to report individual patient data.

\section{Availability of data and material}

The datasets used and/or analysed during the current study are available from the corresponding authors on reasonable request.

\section{Competing interests}

The authors declare no conflicts of interest for the publication of this manuscript.

\section{Funding}

This work was supported by National Natural Science Foundation of China (Grant No. 81602040, 81903067 and 81402341), Clinical science and technology innovation project of Shanghai (SHDC12016104) and Shanghai Science and Technology Committee Project (17411951300). The 
funding bodies had no role in the design of the study and collection, analysis, and interpretation of data and in the writing of the manuscript.

\section{Authors' contributions}

Dr Yang Lv, QingYang Feng, ZhiYuan Zhang and Peng Zheng analyzed and interpreted the patient data. De-Xiang Zhu collected the clinical data, and Yang Lv was a major contributor in writing the manuscript. Pro JianMin Xu and Pro GuoDong He contributed to the design of the work and were the corresponding authors in this manuscript. Dr YuQiu Xu, YiHao Mao and MeiLing Ji provided the research background and perspective views. Pro GuoDong He and Pro JianMin Xu were the corresponding authors and approved the final version of this manuscript to be published.

\section{Acknowledgements}

This manuscript has not been submitted to any other journal and is not currently being considered by another journal for publication. We Thank all the doctors and nurses during the treatment process. Specially, we thank Dr YuXiang Luo and Dr XiaoXiao Liu (Shanghai institute of nutrition and health, Chinese academy of science) for their kind contribution to study design and bioinformatics guidance.

\section{Antibodies}

Antibodies composed of Rabbit anti-human Glutathione Peroxidase 4 (GPX4, Abcam Cat\#ab125066, RRID: AB_10973901), Rabbit anti-human NOX1 (Abcam Cat\# ab78016, RRID: AB_1566505), Rabbit antihuman FACL4 (Abcam Cat\# ab155282, RRID: AB_2714020), Rabbit anti-CD4 (Abcam Cat\# ab183685, RRID: AB_2686917), Rabbit anti-CD8 (Abcam Cat\# ab93278, RRID: AB_10563532) and Rabbit anti-CD86 (Abcam Cat\# ab119857, RRID: AB_10902800).

\section{References}

1. Siegel RL, Miller KD, Goding Sauer A, Fedewa SA, Butterly LF, Anderson JC, et al. Colorectal cancer statistics, 2020. CA Cancer J Clin. 2020;70(3):145-64.

2. Siegel RL, Miller KD, Jemal A. Cancer statistics, 2020. CA Cancer J Clin. 2020;70(1):7-30.

3. Chen W, Zheng R, Baade PD, Zhang S, Zeng H, Bray F, et al. Cancer statistics in China, 2015. CA Cancer J Clin. 2016;66(2):115-32.

4. Oliphant R, Nicholson GA, Horgan PG, Molloy RG, McMillan DC, Morrison DS, et al. Contribution of surgical specialization to improved colorectal cancer survival. Br J Surg. 2013;100(10):1388-95.

5. Conrad M, Angeli JP, Vandenabeele P, Stockwell BR. Regulated necrosis: disease relevance and therapeutic opportunities. Nat Rev Drug Discov. 2016;15(5):348-66.

6. Mou Y, Wang J, Wu J, He D, Zhang C, Duan C, et al. Ferroptosis, a new form of cell death: opportunities and challenges in cancer. J Hematol Oncol. 2019;12(1):34. 
7. Lu B, Chen XB, Ying MD, He QJ, Cao J, Yang B. The Role of Ferroptosis in Cancer Development and Treatment Response. Front Pharmacol. 2017;8:992.

8. Nagpal A, Redvers RP, Ling X, Ayton S, Fuentes M, Tavancheh E, et al. Neoadjuvant neratinib promotes ferroptosis and inhibits brain metastasis in a novel syngeneic model of spontaneous HER2(+ve) breast cancer metastasis. Breast Cancer Res. 2019;21(1):94.

9. Benson AB, Venook AP, Al-Hawary MM, Cederquist L, Chen YJ, Ciombor KK, et al. Rectal Cancer, Version 2.2018, NCCN Clinical Practice Guidelines in Oncology. J Natl Compr Canc Netw. 2018;16(7):874-901.

10. Diagnosis, Treatment Guidelines For Colorectal Cancer Working Group C. Chinese Society of Clinical Oncology (CSCO) diagnosis and treatment guidelines for colorectal cancer 2018 (English version). Chin J Cancer Res. 2019;31(1):117-34.

11. Osterman E, Mezheyeuski A, Sjoblom T, Glimelius B. Beyond the NCCN Risk Factors in Colon Cancer: An Evaluation in a Swedish Population-Based Cohort. Ann Surg Oncol. 2020;27(4):1036-45.

12. Xu J, Fan J, Qin X, Cai J, Gu J, Wang S, et al. Chinese guidelines for the diagnosis and comprehensive treatment of colorectal liver metastases (version 2018). J Cancer Res Clin Oncol. 2019;145(3):725-36.

13. Maag JLV. gganatogram: An R package for modular visualisation of anatograms and tissues based on ggplot2. F1000Res. 2018;7:1576.

14. Jia JB, Wang WQ, Sun HC, Zhu XD, Liu L, Zhuang PY, et al. High expression of macrophage colonystimulating factor-1 receptor in peritumoral liver tissue is associated with poor outcome in hepatocellular carcinoma after curative resection. Oncologist. 2010;15(7):732-43.

15. Mao Y, Feng Q, Zheng P, Yang L, Zhu D, Chang W, et al. Low tumor infiltrating mast cell density confers prognostic benefit and reflects immunoactivation in colorectal cancer. Int $\mathrm{J}$ Cancer. 2018;143(9):2271-80.

16. Ji M, Feng Q, He G, Yang L, Tang W, Lao X, et al. Silencing homeobox C6 inhibits colorectal cancer cell proliferation. Oncotarget. 2016;7(20):29216-27.

17. Subramanian A, Tamayo P, Mootha VK, Mukherjee S, Ebert BL, Gillette MA, et al. Gene set enrichment analysis: a knowledge-based approach for interpreting genome-wide expression profiles. Proc Natl Acad Sci U S A. 2005;102(43):15545-50.

18. Hassannia B, Vandenabeele P, Vanden Berghe T. Targeting Ferroptosis to Iron Out Cancer. Cancer Cell. 2019;35(6):830-49.

19. Tang Z, Li C, Kang B, Gao G, Li C, Zhang Z. GEPIA: a web server for cancer and normal gene expression profiling and interactive analyses. Nucleic Acids Res. 2017;45(W1):W98-W102.

20. Dixon SJ, Lemberg KM, Lamprecht MR, Skouta R, Zaitsev EM, Gleason CE, et al. Ferroptosis: an irondependent form of nonapoptotic cell death. Cell. 2012;149(5):1060-72.

21. Xie Y, Hou W, Song X, Yu Y, Huang J, Sun X, et al. Ferroptosis: process and function. Cell Death Differ. 2016;23(3):369-79. 
22. Shen Z, Song J, Yung BC, Zhou Z, Wu A, Chen X. Emerging Strategies of Cancer Therapy Based on Ferroptosis. Adv Mater. 2018;30(12):e1704007.

23. Zhao Y, Li Y, Zhang R, Wang F, Wang T, Jiao Y. The Role of Erastin in Ferroptosis and Its Prospects in Cancer Therapy. Onco Targets Ther. 2020;13:5429-41.

24. Chen MS, Wang SF, Hsu CY, Yin PH, Yeh TS, Lee HC, et al. CHAC1 degradation of glutathione enhances cystine-starvation-induced necroptosis and ferroptosis in human triple negative breast cancer cells via the GCN2-elF2alpha-ATF4 pathway. Oncotarget. 2017;8(70):114588-602.

25. Murphy MP. Metabolic control of ferroptosis in cancer. Nat Cell Biol. 2018;20(10):1104-5.

26. Sui X, Zhang R, Liu S, Duan T, Zhai L, Zhang M, et al. RSL3 Drives Ferroptosis Through GPX4 Inactivation and ROS Production in Colorectal Cancer. Front Pharmacol. 2018;9:1371.

27. Fan Z, Wirth AK, Chen D, Wruck CJ, Rauh M, Buchfelder M, et al. Nrf2-Keap1 pathway promotes cell proliferation and diminishes ferroptosis. Oncogenesis. 2017;6(8):e371.

28. Feng Q, Chang W, Mao Y, He G, Zheng P, Tang W, et al. Tumor-associated Macrophages as Prognostic and Predictive Biomarkers for Postoperative Adjuvant Chemotherapy in Patients with Stage II Colon Cancer. Clin Cancer Res. 2019;25(13):3896-907.

29. Cole-Ezea P, Swan D, Shanley D, Hesketh J. Glutathione peroxidase 4 has a major role in protecting mitochondria from oxidative damage and maintaining oxidative phosphorylation complexes in gut epithelial cells. Free Radic Biol Med. 2012;53(3):488-97.

30. Hangauer MJ, Viswanathan VS, Ryan MJ, Bole D, Eaton JK, Matov A, et al. Drug-tolerant persister cancer cells are vulnerable to GPX4 inhibition. Nature. 2017;551(7679):247-50.

31. Doll S, Proneth B, Tyurina YY, Panzilius E, Kobayashi S, Ingold I, et al. ACSL4 dictates ferroptosis sensitivity by shaping cellular lipid composition. Nat Chem Biol. 2017;13(1):91-8.

32. Dachert J, Ehrenfeld V, Habermann K, Dolgikh N, Fulda S. Targeting ferroptosis in rhabdomyosarcoma cells. Int J Cancer. 2020;146(2):510-20.

33. Elliott MR, Ravichandran KS. The Dynamics of Apoptotic Cell Clearance. Dev Cell. 2016;38(2):147-60.

34. Zitvogel L, Kroemer G. Interferon-gamma induces cancer cell ferroptosis. Cell Res. 2019;29(9):692-3.

35. Wang W, Green M, Choi JE, Gijon M, Kennedy PD, Johnson JK, et al. CD8(+) T cells regulate tumour ferroptosis during cancer immunotherapy. Nature. 2019;569(7755):270-4.

36. Huang Y, Tian C, Li Q, Xu Q. TET1 Knockdown Inhibits Porphyromonas gingivalis LPS/IFN-gammaInduced M1 Macrophage Polarization through the NF-kappaB Pathway in THP-1 Cells. Int J Mol Sci. 2019;20(8).

\section{Figures}



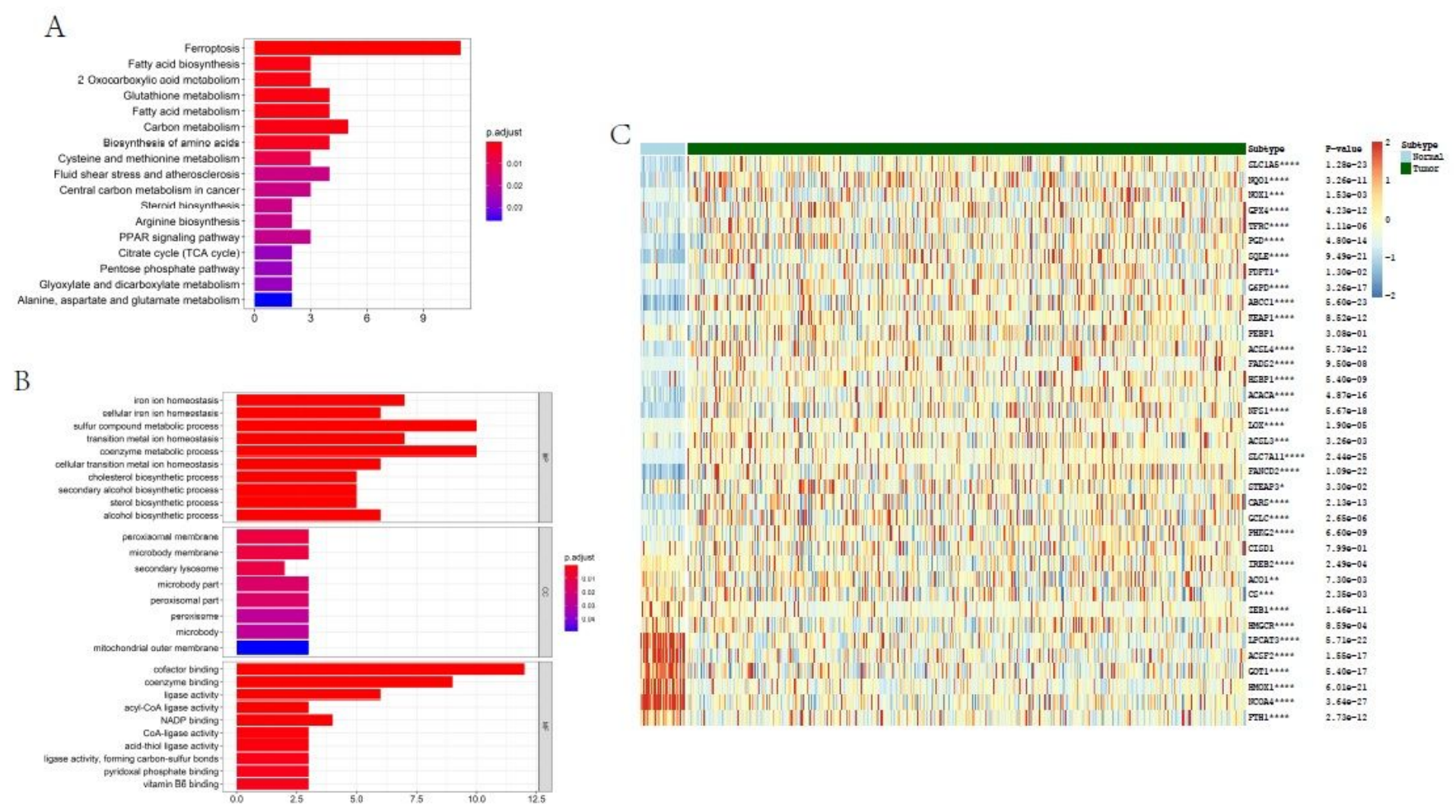

D
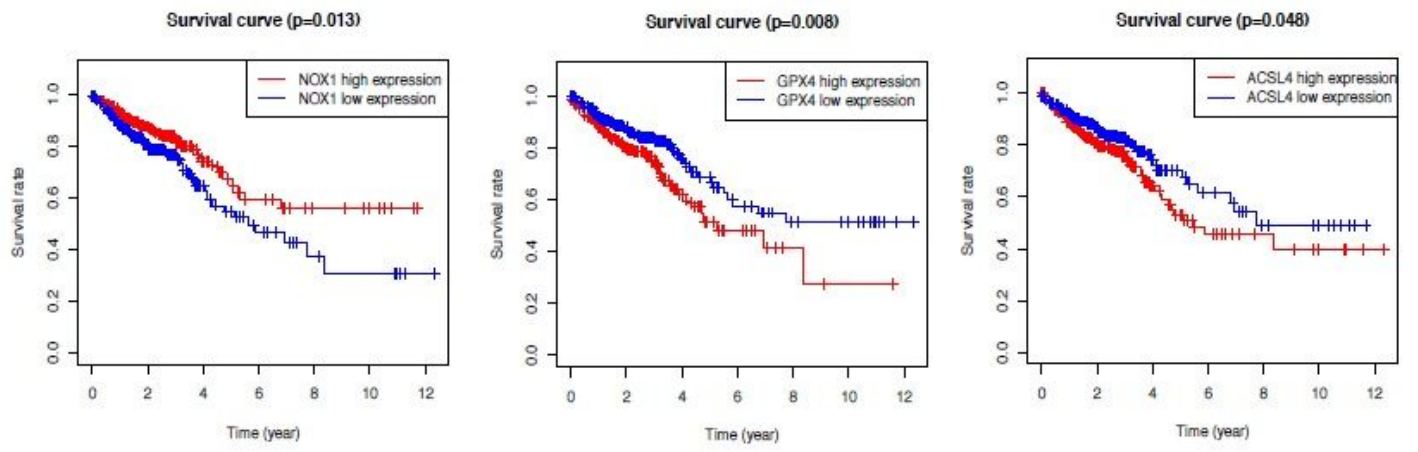

Figure 1

(A) KEGG analysis confirmed the key pathways for selected 42 genes and ferroptosis ranked the top pathway; (B) GO analysis revealed potential cellular component (CC), molecular function (MF) and biological process (BP); (C) mRNA expression difference heatmap of 42 genes between CRC tumor and normal epithelium; (D) Kaplan-meier analysis of OS for GPX4, NOX1 and ACSL4. Abbreviation: KEGG, Kyoto Encyclopedia of Genes and Genomes; GO, Gene ontology; CRC, colorectal cancer; OS, overall survival. 

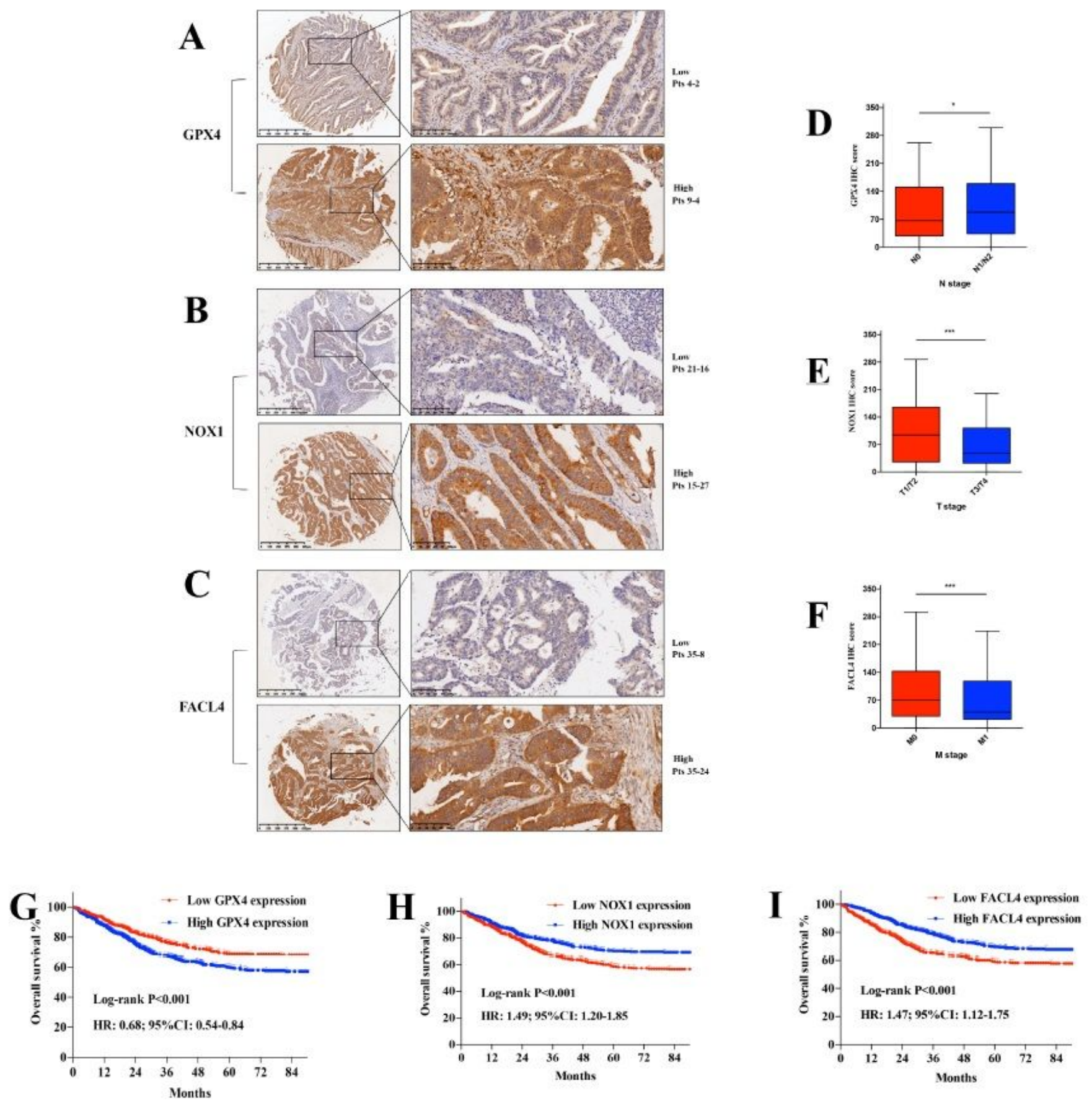

\section{Figure 2}

(A) Representative images of IHC staining for GPX4; (B) Representative images of IHC staining for NOX1;

(C) Representative images of IHC staining for FACL4; (D) IHC score between N0 stage and N1/N2 stage; (E) IHC score between T1/T2 stage and T3/T4 stage; (F) IHC score between M0 stage and M1 stage; (G) Kaplan-meier analysis of OS for GPX4; (H) Kaplan-meier analysis of OS for NOX1; (I) Kaplan-meier analysis of OS for FACL4. Abbreviation: IHC, immunohistochemistry; CRC, colorectal cancer; OS, overall survival. 

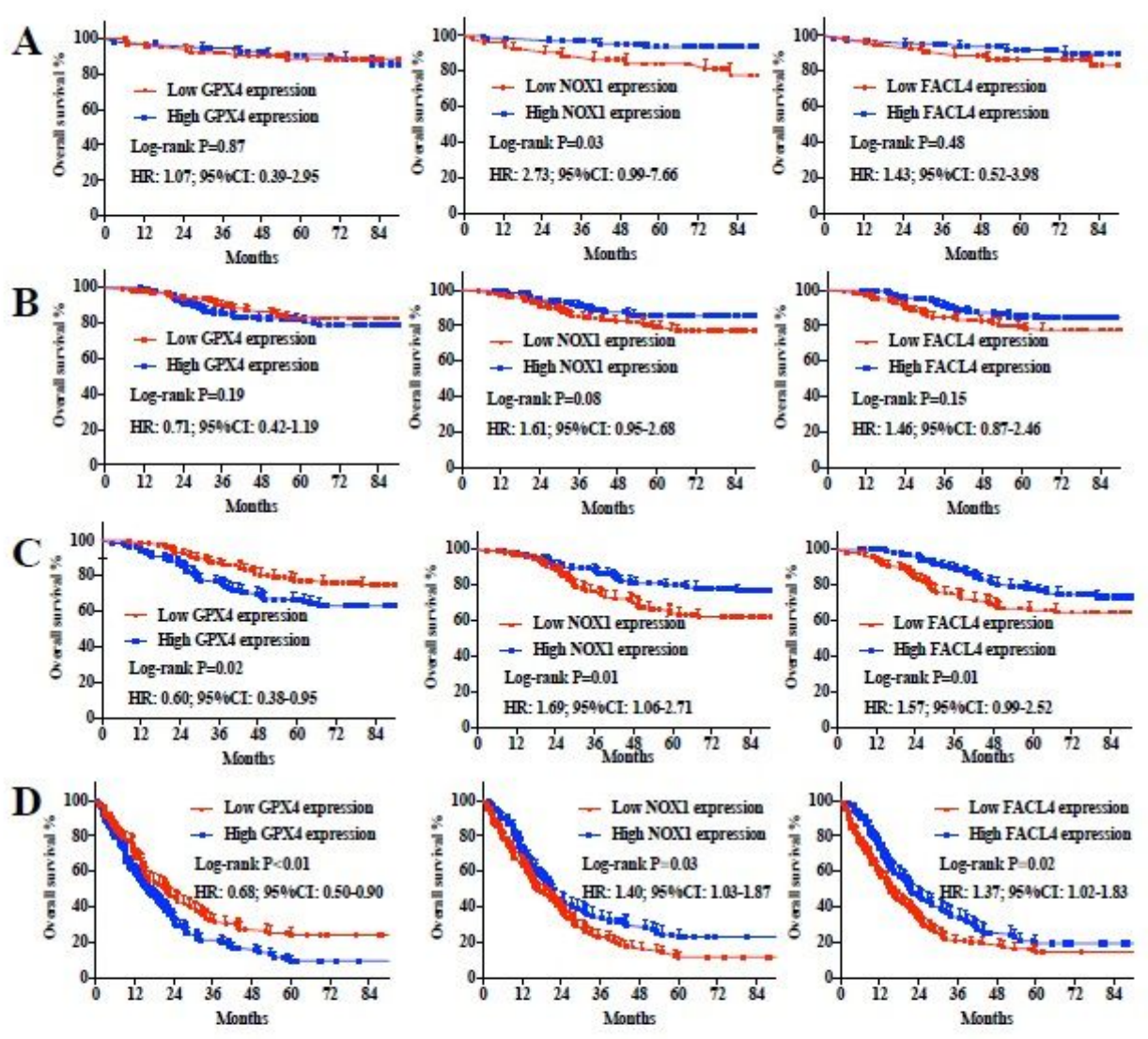
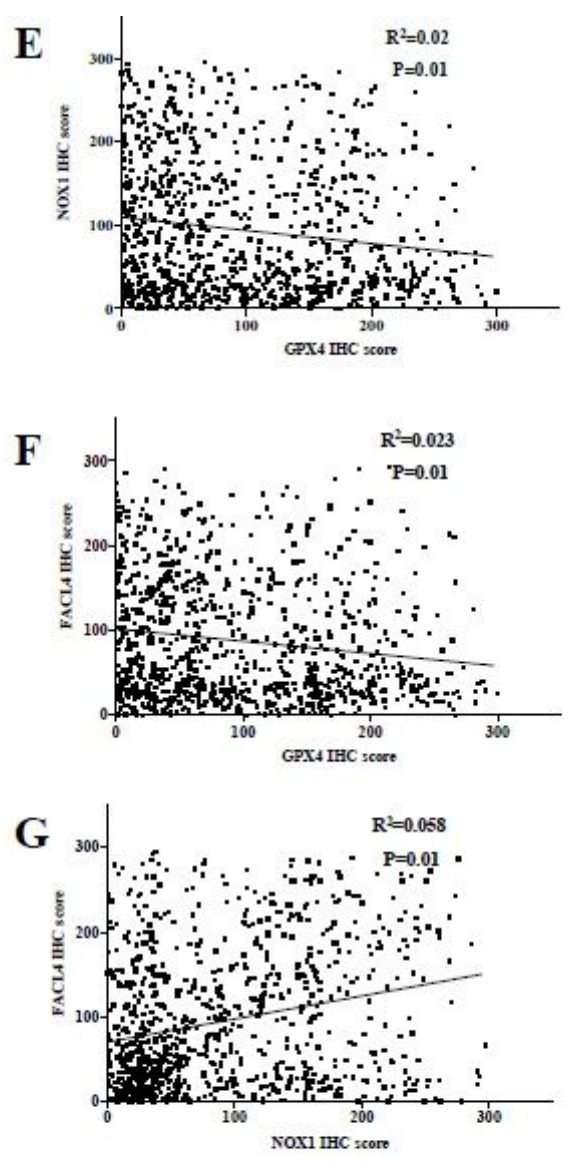

H Points $\quad$\begin{tabular}{lllllllllll}
0 & 1 & 2 & 3 & 4 & 5 & 6 & 7 & 8 & 9 & 10 \\
\hline & & & &
\end{tabular}

GPX4

NOX1

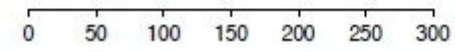

FACL4

$$
300100
$$

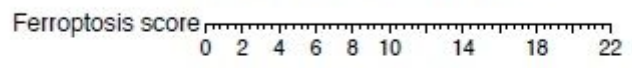

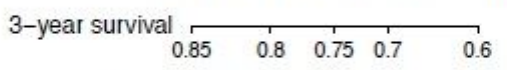

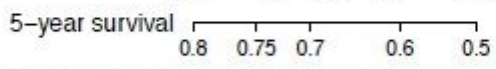

7-year survival
I
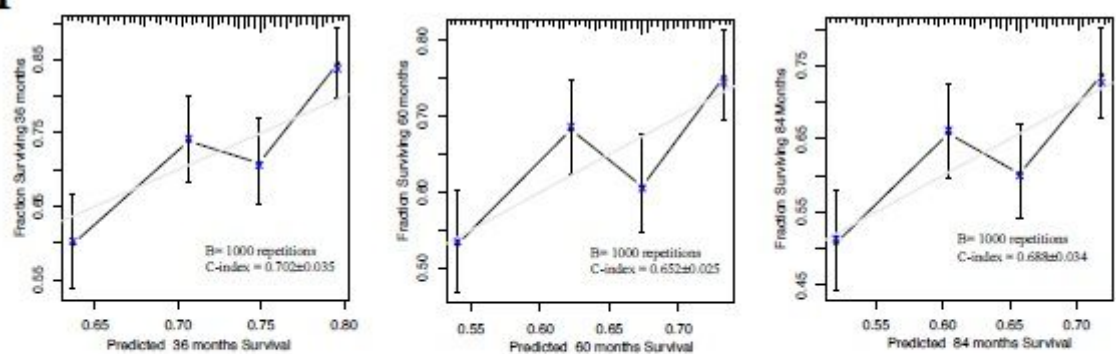

\section{Figure 3}

Kaplan-meier analysis of GPX4, NOX1 and FACL4 expression on OS in stage I (A), stage II (B), stage III (C) and stage IV (D); (E) IHC score correlation between GPX4 and NOX1; (F) IHC score correlation between GPX4 and FACL4; (G) IHC score correlation between FACL4 and NOX1; $(\mathrm{H})$ Construction of ferroptosis score based on Cox analysis; (I) Validation of ferroptosis score on 3 years, 5 years and 7 years' survival rate based on validation cohort data. Abbreviation: IHC, immunohistochemistry; CRC, colorectal cancer; OS, overall survival. 

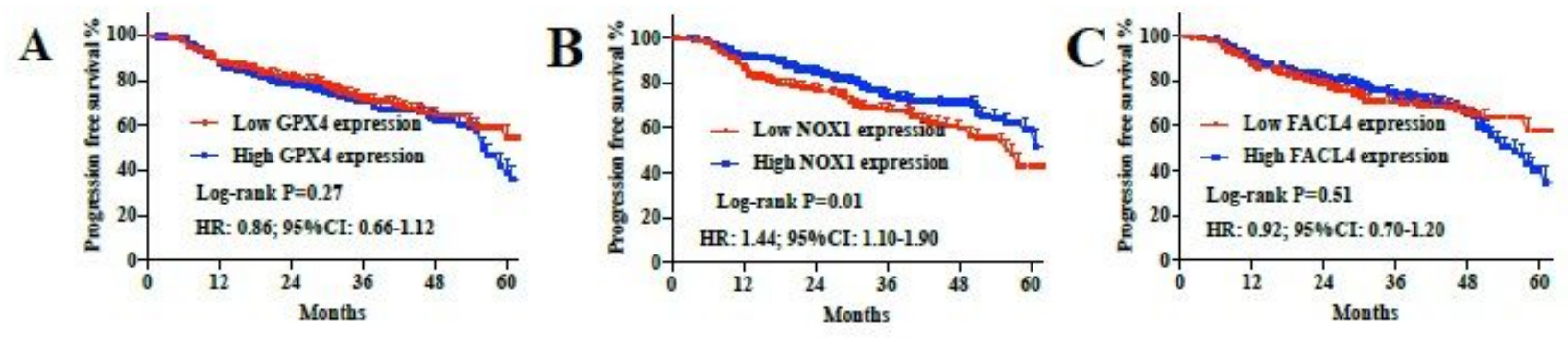

D

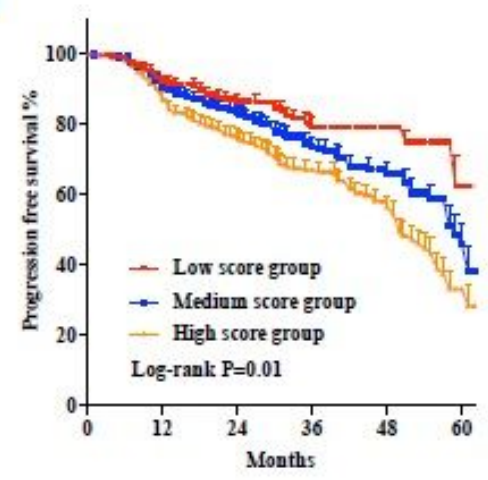

$\mathbf{E}$

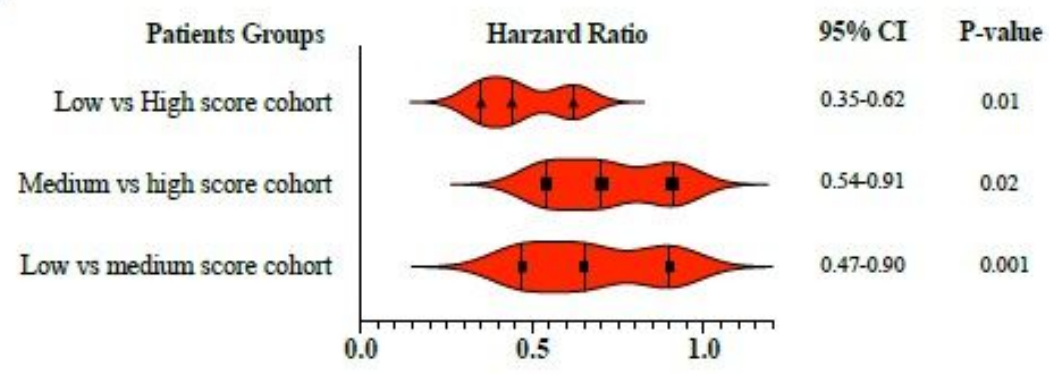

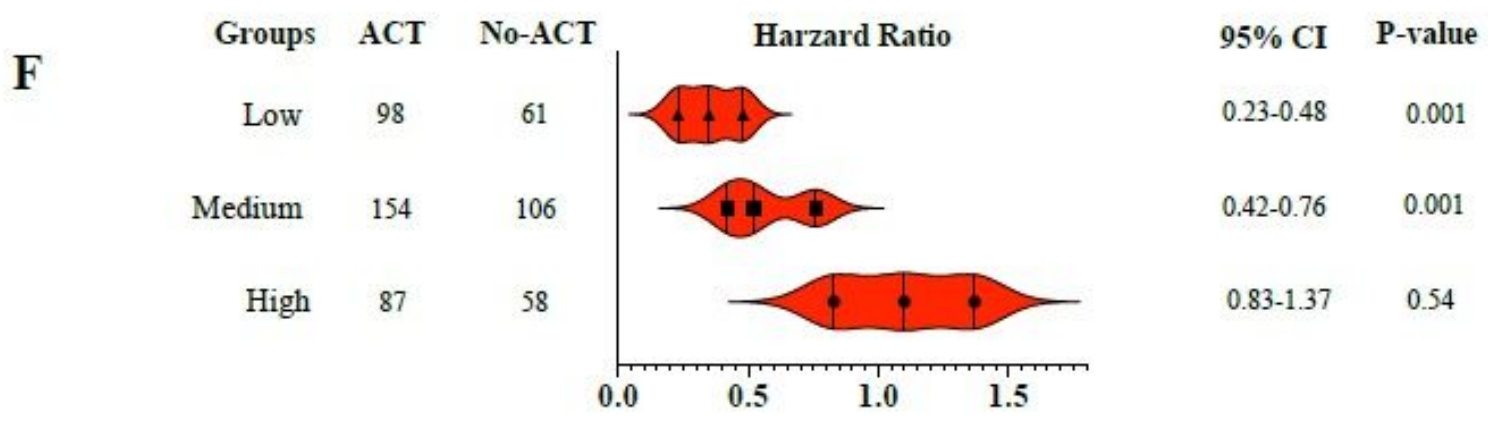

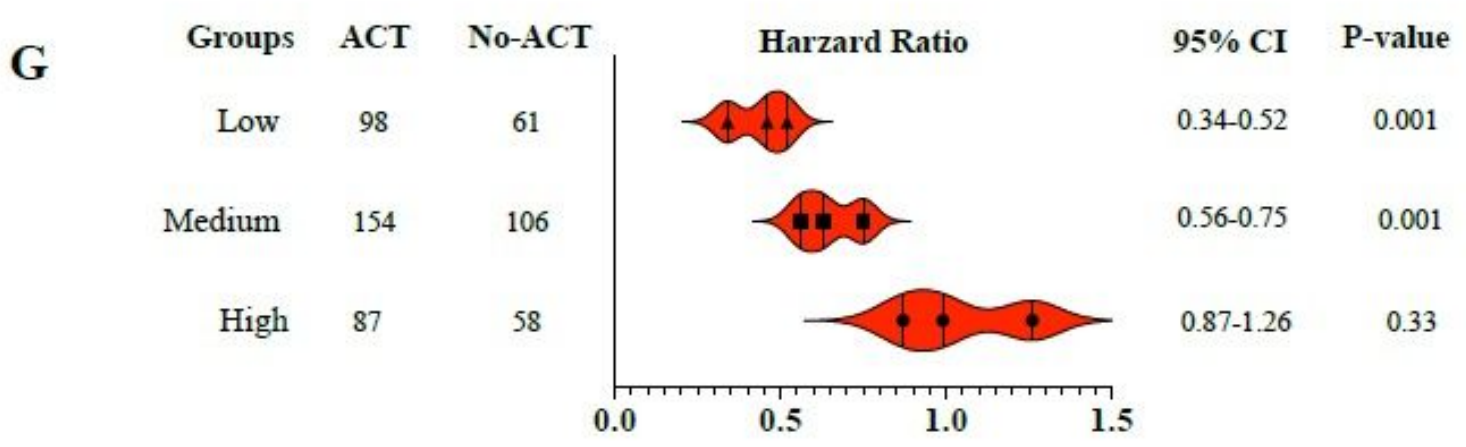

\section{Figure 4}

Kaplan-meier analysis of GPX4 (A), NOX1 (B) and FACL4 (C) expression on PFS; (D) Kaplan-meier analysis of ferroptosis score on PFS stratification; (E) Cox proportional hazards regression analysis for comparisons of PFS in different risk groups; (F) Cox proportional hazards regression PFS analysis for the difference of responsiveness to ACT within different risk groups; (G) Cox proportional hazards regression 
OS analysis for the difference of responsiveness to ACT within different risk groups. Abbreviation: PFS, progression free survival; CRC, colorectal cancer; OS, overall survival; ACT, adjuvant chemotherapy.

A
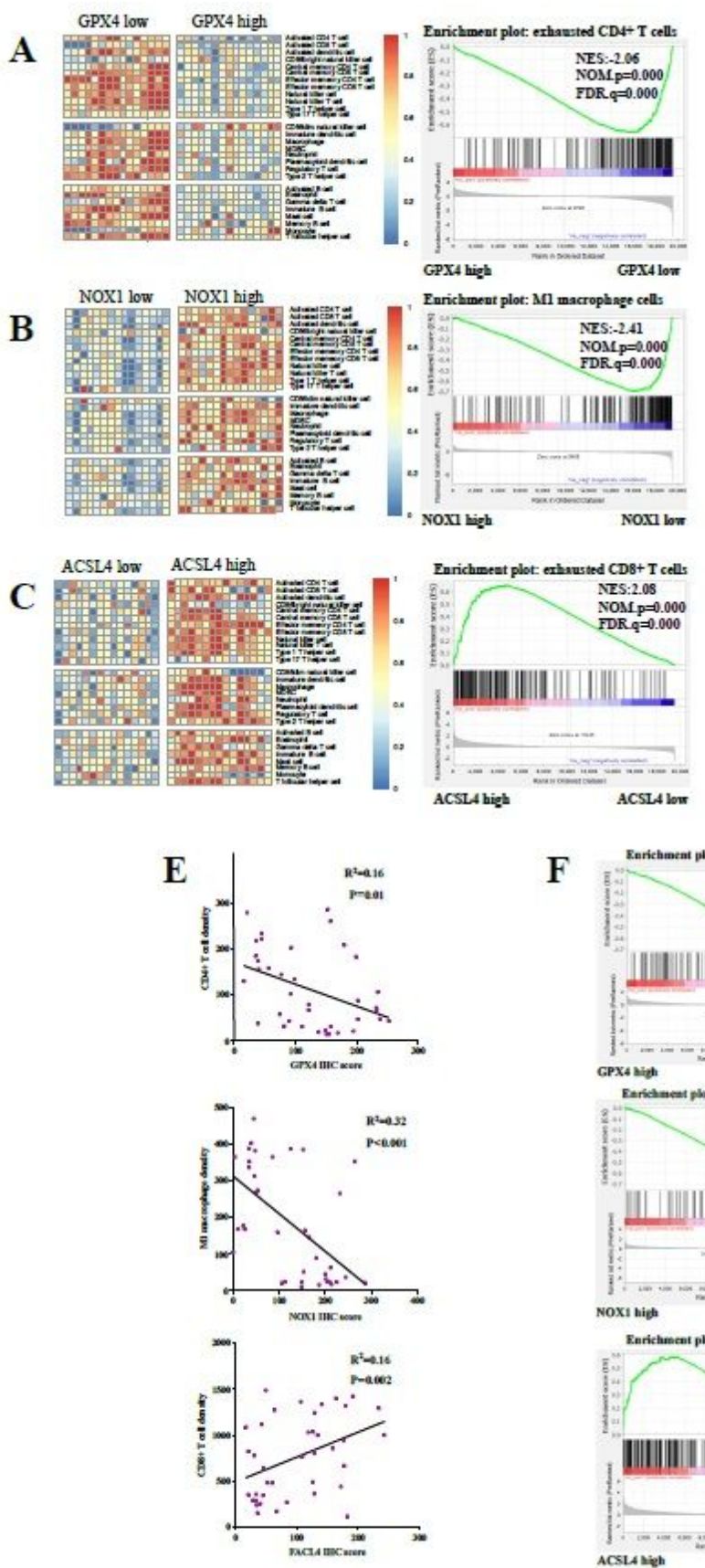

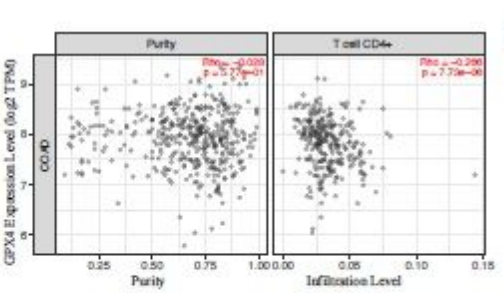

D
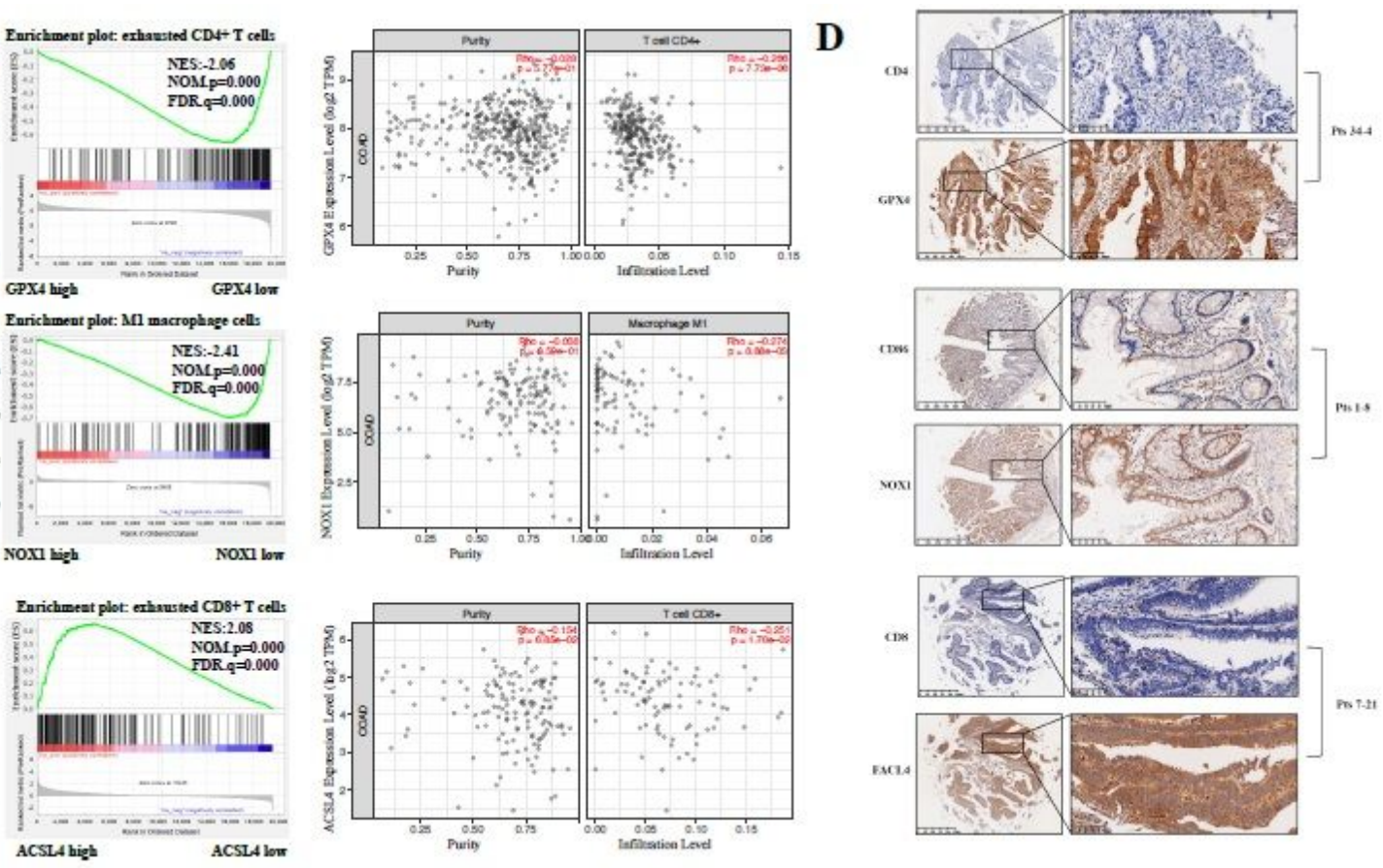

\section{Figure 5}

(A) GSEA analysis for GPX4 expression and CD4+ T cell infiltration; (B) GSEA analysis for NOX1 expression and M1 macrophage infiltration; (C) GSEA analysis for ACSL4 expression and CD8+ $T$ cell infiltration; (D) Representative images of double staining of CD4 and GPX4 (top) or CD86 and NOX1 (medium) or CD8 and FACL4 (bottom) on TMA; (E) scatter diagram between ferroptosis markers and immune infiltration; (F) GSEA analysis for hallmarks of cancer on GPX4, NOX1 and ACSL4 mRNA expression; (G) scatter diagram between ferroptosis markers and downstream of IFN-Y (JAK and Stat1). Abbreviation: GSEA, gene set enrichment analysis; TMA, tissue microarray. 

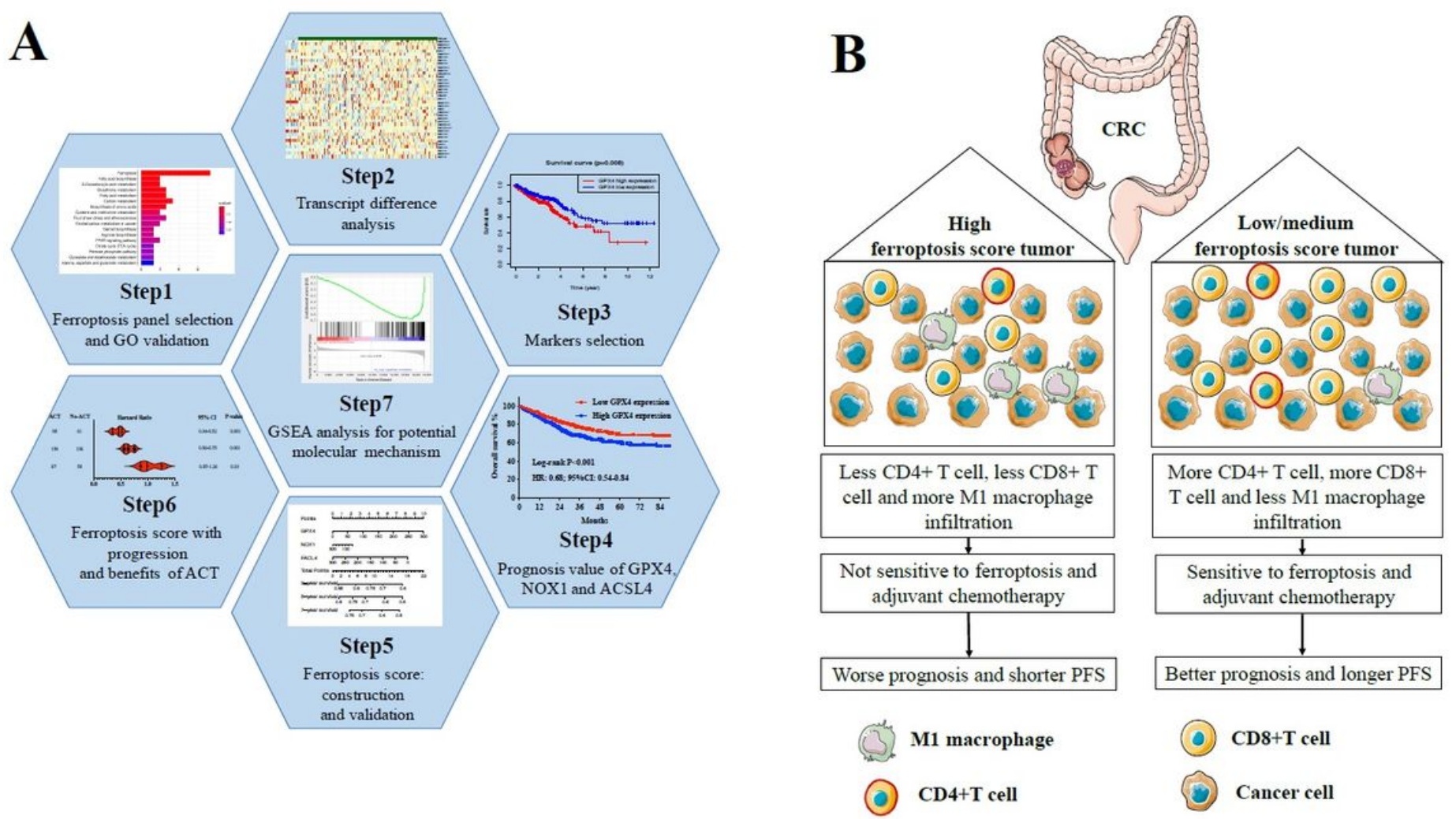

Figure 6

(A) Graphical summary of this study and Schematics depicting the materials and methods used in the research; (B) the prognostic role of ferroptosis and the underlying immune-activation functions on CRC. Abbreviation: CRC, colorectal cancer.

\section{Supplementary Files}

This is a list of supplementary files associated with this preprint. Click to download.

- Supplementarymaterials.docx 